\title{
Heart Rate Variability Analysis in Risk of Asthma Stratification
}

\author{
Javier Milagro ${ }^{1,2}$, Eduardo Gil ${ }^{1,2}$, Jesús Lázaro ${ }^{3,4}$, Ville-Pekka Seppä ${ }^{5}$, \\ L. Pekka Malmberg ${ }^{6}$, Anna S. Pelkonen ${ }^{6}$, Anne Kotaniemi-Syrjänen ${ }^{6}$, \\ Mika J. Mäkelä ${ }^{6}$ Jari Viik ${ }^{5}$, Raquel Bailón ${ }^{1,2}$ \\ ${ }^{1}$ Biomedical Signal Interpretation and Computational Simulation (BSICoS) \\ Instituto de Investigación en Ingeniería de Aragón (I3A) \\ Universidad de Zaragoza, Mariano Esquillor s/n, 50018, Zaragoza, Spain. \\ Tel.+34-976762707, e-mail: milagro@unizar.es \\ ${ }^{2}$ CIBER-BBN: Centro de Investigación Biomédica en Red - Bioingeniería, Biomateriales y Nanomedicina \\ ${ }^{3}$ KU Leuven, Leuven, Belgium \\ ${ }^{4}$ IMEC, Leuven, Belgium \\ ${ }^{5}$ Tampere University of Technology, Tampere, Finland \\ ${ }^{6}$ Helsinki University Hospital, Helsinki, Finland
}

\begin{abstract}
Early diagnosis of asthma is crucial to avoid longterm effects such as permanent airway obstruction. Pathogenesis of asthma has been related with autonomic nervous system (ANS) dysfunction, concretely with abnormal parasympathetic activity. As heart rate variability (HRV) analysis does reflect ANS activity, it has been employed here in risk of asthma stratification.
\end{abstract}

\section{Introduction}

Asthma is a chronic disease that inflames and narrow the airways producing a difficulty for breathing. It usually starts during childhood and its incidence and prevalence have augmented in recent years [1]. Despite this, there is still not a method for its early diagnosis, and classical spirometry studies could result invasive for young children. Although most of the symptoms of asthma can be controlled using appropiate medication, it is important to diagnose and start to control it as soon as possible in order to prevent patients from permanent airway obstruction.

Several studies have pointed to autonomic dysfunction as playing an important role in the pathogenesis of asthma, given the fact that parasympathetic nervous system (PNS) does exert a control over bronchoconstriction [2] and broncomotor mechanisms [3]. As PNS activity is reflected in the high frequency (HF) component of heart rate variability (HRV) spectrum, HRV analysis has been used in the assessment of increased vagal tone in asthmatics [4].
On the other hand, asthma has been described as a state of increased regularity attending to reduced complexity of the airflow pattern in asthmatics [5].

As respiratory modulation of HRV is also driven by PNS a novel index combining information from HRV spectrum and respiration is studied here together with other classical time and frequency domain HRV indexes.

\section{Materials and Methods}

\section{Study population}

The data base employed here is formed by 27 preschool children from who electrocardiogram (ECG) and impedance pneumography (IP) signals were acquiered. Children were classified in high- or low-risk (HiR and LoR respectively) of developing asthma attending to their modified asthma predictive index (mAPI) [6]. Data acquisition was approved by an institutional pediatric ethics review board and informed written parental consent was received.

\section{HRV analysis}

Both ECG and IP signals were first conveniently preprocessed. Afterwards, RR interval series were derived from these preprocessed ECG signals, and they were further used for obtaining the HRV spectrum. Classical time domain indexes were derived from the RR interval series: NN, SDNN, SDSD, RMSSD and pNN50, while classical 
frequency domain indexes were obtained from the HRV spectrum: low frequency (LF) power, HF power, LF to HF power ratio and normalized LF power [7].

Afterwards, a novel index that combines information from HRV and IP is proposed. It is based in the analysis of the HF band of HRV spectrum, including respiratory information. The aim of this index is to characterize the differences that may exist between the different groups in a non-invasive way. Moreover, the possibility of employing only the ECG signal or either the IP signal is considered.

\section{Results}

Statistical significant differences were found between HiR and LoR. The results suggest a distinct behaviour of the PNS attending to the risk of developing asthma, being consistent with previous studies that considered HRV analysis [4] or airflow measurements analysis [5,7]. Although previous classification of the degree of asthma had been performed in young children [4], to the authors knowledge no studies with pre-school children had been performed before.

The possibility of employing either the ECG or the IP signal for distinguishing between groups suggest that non-invasive ways of diagnosing asthma in its earliest stages should be further studied, so that invasive procedures such as spirometries (that are not invasive for adults, but might be for young children) or bronchial biopsy can be avoided.

\section{Conclusions}

HRV analysis has been employed in the characterization of ANS activity of pre-school children that had been classified in function of their asthma risk. Differences between groups support the use of HRV as a helpful complement for the study of the mechanisms underlying asthma. The noninvasive characteristic of HRV analysis makes it especially suitable for children, as classical spirometries could result intrusive for them.

\section{Acknowledgments}

This work was supported by grant BES-2015073694 and project TIN2014-53567-R from Ministerio de Economía y Competitividad. Also by Government of Aragón and European Social Fund (EU) through Grupo Consolidado BSICoS (T96), by CIBER in Bioengineering, Biomaterials \& Nanomedicine (CIBER-BBN) through Instituto de Salud Carlos III, by Helsinki University Research Grants, by Sigrid Juselius Foundation and by Tampere Tuberculosis Foundation.

\section{REFERENCES}

[1]. SHEFFER, A. L., et al. International consensus report on diagnosis and treatment of asthma. European Respiratory Journal, 1992, vol. 5, no 5, p. 601-641.

[2]. LEWIS, M. J.; SHORT, A. L.; LEWIS, K. E. Autonomic nervous system control of the cardiovascular and respiratory systems in asthma. Respiratory medicine, 2006, vol. 100, no 10, p. 1688-1705.

[3]. MORRISON, J. F. J.; PEARSON, S. B.; DEAN, H. G. Parasympathetic nervous system in nocturnal asthma. British medical journal (Clinical research ed.), 1988, vol. 296, no 6634, p. 1427.

[4]. EMIN, O., et al. Autonomic nervous system dysfunction and their relationship with disease severity in children with atopic asthma. Respiratory physiology \& neurobiology, 2012, vol. 183, no 3, p. 206-210.

[5]. VEIGA, J., et al. Approximate entropy as a measure of the airflow pattern complexity in asthma. In Engineering in Medicine and Biology Society (EMBC), 2010 Annual International Conference of the IEEE. IEEE, 2010. p. 2463-2466.

[6]. GUILBERT, T. W., et al. Atopic characteristics of children with recurrent wheezing at high risk for the development of childhood asthma. Journal of Allergy and Clinical Immunology, 2004, vol. 114, no 6, p. 1282-1287.

[7]. TASK FORCE OF THE EUROPEAN SOCIETY OF CARDIOLOGY, et al. Heart rate variability standards of measurement, physiological interpretation, and clinical use. Eur heart J, 1996, vol. 17, p. 354-381.

[8]. QUE, C-L., et al. Homeokinesis and short-term variability of human airway caliber. Journal of Applied Physiology, 2001, vol. 91, no 3, p. 1131-11 